\title{
EXPORTAÇÃO BRASILEIRA DE PAPEL E CELULOSE: SUA DINÂMICA PELA EQUAÇÃO GRAVITACIONAL ${ }^{1}$
}

Thiago Taglialegna Salles², Márcio Lopes da Silva ${ }^{3}$, Naisy Silva Soares ${ }^{4}$ e Angelo Casali de Moraes $^{5}$

\begin{abstract}
RESUMO - O Brasil figura entre os principais produtores de celulose e papel e a indústria brasileira é mundialmente competitiva no setor. Com interesses voltados para este cenário, aplicou-se neste estudo a equação de gravidade, usada para explicar de maneira empírica fluxos de comércio entre países, com objetivo de analisar a dinâmica da exportação de papel e de celulose pelo Brasil no período de 1997 a 2005. O modelo gravitacional foi adaptado e suas variáveis explicativas foram: PIB do Brasil, PIB do país importador, PIB per capita do país importador e distância entre o importador e o Brasil. Os resultados mostraram que a distância influenciou negativamente as exportações de papel, mas ao contrário do esperado, seu coeficiente associado apresentou valor positivo para a exportação de celulose. O PIB per capta dos países importadores teve contribuição positiva para as exportações de celulose e negativa para as de papel. Tanto o PIB do Brasil, quanto o PIB dos países importadores tiveram seus coeficientes associados apresentando valor positivo, como esperado. Concluiu-se que o modelo obteve êxito na predição dos fluxos bilaterais do comércio das commodities em análise. As exportações, tanto de papel quanto de celulose, foram mais sensíveis a variações no PIB do Brasil do que a variações no PIB, PIB per capita e na distância dos países importadores. A distância influencia negativamente as exportações de papel, mas não as da celulose brasileira.
\end{abstract}

Palavras-chave: Exportação de papel e celulose, Modelo gravitacional e Brasil.

\section{BRAZILIAN EXPORTS OF WOOD PULP AND PAPER: ITS DYNAMICS BY THE GRAVITATIONAL EQUATION}

\begin{abstract}
Brazil is among the leading producers of wood pulp and paper and the Brazilian industry is globally competitive in this segment. With interest toward this scenario, this study applied the gravity equation, used to empirically explain trade flows between countries, to analyze the dynamics of wood pulp and paper exports from Brazil from 1997 to 2005 . The gravity equation was modified and its explanatory variables were: Brazil's GDP, the GDP of the importing country, GDP per capita of the importing country and the distance from the importer country and Brazil. The results showed that distance negatively affected papel exports, but contrary to expectations, its associated coefficient had a positive value for wood pulp export. The GDP per capita of the importing countries positively contributed to wood pulp exports and it negatively contributed to paper exports. Brazil's GDP and the GDP of the importing countries as well had their associated coefficients with a positive value, as expected. It was concluded that the model succeeded in predicting the commercial bilateral flows of the analyzed commodities. Exports of paper and wood pulp as well, were more sensitive to changes in Brazil's GDP than to changes in GDP, GDP per capita and distance from importing countries. The distance negatively influences exports of paper, but not the Brazilian exports of wood pulp.
\end{abstract}

Keywords: Brazil, Gravity model, Paper and Wood pulp export.

\footnotetext{
${ }^{1}$ Recebido em 25.08.2009 e aceito para publicação em 14.04.2011.

${ }^{2}$ DAP Engenharia Florestal, DAP Florestal, Brasil. E-mail: <emaildothiago2006@yahoo.com.br>.

${ }^{3}$ Universidade Federal de Viçosa, UFV, Brasil. E-mail: <marlosil@ufv.br>.

${ }^{4}$ Universidade Estadual de Santa Cruz, UESC, Brasil. E-mail: <naisysilva@yahoo.com.br>.

${ }^{5}$ Programa de Pós-Graduação em Ciencia Florestal da Universidade Federal de Viçosa, UFV, Brasil. E-mail: <angelo.moraes@ufv.br>.
} 


\section{INTRODUÇÃO}

Em 2008, o comércio exterior brasileiro alcançou a cifra de US\$371,1 bilhões e o saldo comercial atingiu US\$ 24,7 bilhões. As exportações encerraram o ano de 2008 com valor inédito de US\$ 197,9 bilhões, apresentando um crescimento de 23,2\% em relação ao ano anterior. Estes números indicam o prosseguimento do nível de abertura da economia e a maior inserção do Brasil no comércio mundial. (MDIC, 2008).

O setor florestal tem contribuído com parcelas cada vez maiores para a economia brasileira. Seus números expressivos o revelam como alternativa promissora e sustentável para o País, considerando principalmente seu baixo custo ambiental e a grande capacidade de gerar e multiplicar postos de trabalho (VIEIRA et al., 2006). O cenário relacionado à expansão da atividade geradora de exportações encontra-se pouco favorável. No entanto, a agroindústria nacional vem se mantendo como um dos setores mais produtivos e eficientes do país, graças ao seu nível técnico e competitividade externa (RADICCHI, 2004).

Destacam-se, dentre os produtos florestais, a celulose e o papel que tiveram o valor de suas exportações no ano de 2008 igual a US \$ 5,8 bilhões e saldo comercial igual a US $\$ 4,1$ bilhões (16,6\% do Saldo da Balança Comercial do Brasil) proporcionando a manutenção de 114.000 empregos diretos (67 mil na indústria, $47 \mathrm{mil} \mathrm{na}$ floresta), 570 mil empregos indiretos e o pagamento de R \$2,2 bilhões em impostos. Este setor realizou, nos últimos 10 anos, investimentos na ordem de US\$ 12 bilhões, (BRACELPA, 2009). Graças às condições edafoclimáticas favoráveis e grande disponibilidade de terras para cultivo, o Brasil possui condições adequadas ao cultivo do eucalipto e de outras espécies potenciais produtoras de fibras, o que reflete num baixíssimo custo de produção aliado a alta produtividade. Por conseqüência, o país encontra-se entre os maiores produtores mundiais de papel e celulose, com a venda desses produtos para o exterior representando cerca de $4 \%$ das exportações nacionais. (SOUZA; OLIVEIRA, 2002; VALVERDE et al., 2006).

Segundo resultados obtidos por Valverde et al., (2006), em breve, o Brasil poderá ser o maior exportador de celulose em termos de valor exportado. Atualmente a nação é a principal exportadora de celulose de eucalipto, ocupando 46\% do mercado deste produto (RADICCHI, 2004).
No caso dos papéis, o setor em 2007 também obteve bom desempenho e, em comparação com o ano de 2006, indicou um crescimento de $2,8 \%$. A quantidade da commodity que é exportada pelas empresas brasileiras vem do excesso produzido, já que a produção tem como prioridade abastecer o mercado interno. Desta forma, as exportações do produto estão sujeitas a variadas flutuações. Mesmo assim, para o ano de 2008, por exemplo, houve boas expectativas por parte dos produtores, com as fábricas de papel preparadas para produzir 9,2 milhões de toneladas, volume 3,1\% superior ao alcançado em 2007 (RADICCHI, 2004; MORAES, 2008).

Neste cenário de prosperidade, observa-se a importância da análise das variáveis envolvidas na dinâmica do comércio internacional de papel e celulose e a maneira como as mesmas influenciam no seu fluxo. Também se mostram necessários o estudo e a avaliação de métodos para projetar as intensidades de tais fluxos.

A chamada equação gravitacional vem a longo tempo sendo utilizada para descrever de forma empírica os padrões do comércio entre países. Com uma forma funcional que remete à lei da gravidade na física, a equação gravitacional relaciona o comércio entre dois países de forma positiva para suas rendas e negativamente para a distância entre eles (DEARDORFF, 1998). Trabalhos foram feitos com fins de sustentar essa relação empírica com uma base teórica (ANDERSON, 1979; BERGSTRAND, 1985, 1989; EVENETT; KELLER, 2002) e Deardorff (1998) menciona que a equação gravitacional pode ser derivada a partir de qualquer modelo de linha de base de comércio.

Diante do exposto, o presente estudo teve por objetivo analisar os fluxos de exportação de papel e celulose pelo Brasil por meio da adaptação da equação gravitacional, avaliando o seu comportamento em relação ao PIB brasileiro e às variáveis PIB, PIB per capta e distância em relação ao Brasil, referentes aos países importadores das commodities.

\section{MATERIAL E MÉTODOS}

\subsection{Modelo teórico}

Baseada na "lei da gravitação universal" proposta por Isaac Newton, a equação gravitacional descreve a atração entre dois corpos sendo diretamente proporcional ao produto de suas massas e inversamente proporcional à distância entre eles e é representada da seguinte forma (equação 1 ): 


$$
\omega_{i j}=\varphi \frac{M_{i} M_{j}}{D_{i j}}
$$

em que:

$\omega_{i j}=$ atração entre dois corpos $i$ e $j$;

$\omega=$ constante gravitacional;

$M_{i}=$ massa do corpo $i$;

$M_{j}=$ massa do corpo $j \mathrm{e}$

$D_{i j}=$ distância entre os corpos.

A equação (1) pode ser linearizada, gerando um modelo que permite após seu ajuste, a análise do efeito independente de cada variável. A equação gravitacional linearizada fica expressa por (equação 2):

$\ln \omega_{i j}=\beta_{0}+\beta_{1} \ln M_{i}+\beta_{2} \ln M_{j}+\beta_{3} \ln D_{i j}+\varepsilon_{i j} \quad e q .2$

Em seu emprego nos estudos de economia, a variável $\omega_{i j}$ indica o volume de comércio da região $i$ para a região $j, M_{i}$ é o PIB da região exportadora, $M_{j}$ o PIB da região importadora, $D_{i j}$ a distância entre as regiões, $\beta_{i}$ os parâmetros do modelo, $\varepsilon_{i j}$ o erro aleatório e ln o logaritmo neperiano.

Apesar de por um tempo existirem declarações de que o modelo não possui fundamentação teórica, Frankel (1997) diz que há uma revivicação do modelo por pelo menos três motivos: 1) suas fundamentações teóricas terem sido aprimoradas, erguendo-se em sua maioria a partir de teorias modernas sobre comércio de bens substitutos imperfeitos, 2) seu sucesso empírico na predição de fluxos bilaterais de comércio e 3) um novo interesse entre os economistas no ramo da geografia e comércio, que procura tratar países ou regiões como sendo fisicamente instalados em uma localização particular ao invés de entidades indefinidas no espaço.

\subsection{O modelo aplicado}

O modelo empregado neste estudo envolveu, além das variáveis presentes na equação (2), a adição de outras que representam fatores determinantes para descrever mais precisamente as tendências dos fluxos. A forma final foi (equação 3):

$$
\begin{aligned}
& \ln \omega_{i j}=\beta_{0}+\beta_{1} \ln M_{i}+\beta_{2} \ln M_{j}+\beta_{3} \ln D_{i j}+ \\
& +\varepsilon_{i j}+\beta_{3} \ln m_{j}+\sum_{n=5}^{N} \beta_{n} Y_{n i j}+\varepsilon_{i j}
\end{aligned}
$$

Onde as variáveis $\omega_{i j}, M_{i}, M_{j}, D_{i j}$ e $\beta_{i}$ representam as características anteriormente mencionadas e $m_{j}$ é o PIB per capita da região importadora. $Y$ são variáveis dummies binárias associadas aos países. Elas foram inseridas para controlar os efeitos de diferenças bruscas e desmedidas na amplitude dos fluxos de exportação, havendo dificuldade de ajuste na ausência das mesmas. Frankel (1997) descreve a inclusão de variáveis dummy e também do PIB per capita na equação gravitacional como variáveis explicativas para aumentar a eficiência do modelo. Também podem ser encontradas referências a este tipo de abordagem em Almeida e Silva (2006) e Silva et al. (2007).

O modelo foi ajustado para estimar a exportação das commodities celulose e papel, visando identificar a maneira na qual as variáveis independentes influenciam no processo. A forma log-linear foi utilizada, pois apresentou melhor ajuste na pré-análise dos dados e permitiu examinar o efeito das variáveis de forma individual. O método de ajuste foi o de mínimos quadrados ordinários (MQO).

\subsection{Justificativa das variáveis utilizadas}

PIB da região exportadora $\left(M_{i}\right)$ : representa o poder de produção (tamanho econômico) da região exportadora. Espera-se sinal positivo, refletindo o aumento da quantidade exportada à medida que $M_{i}$ aumenta.

PIB da região importadora $\left(M_{j}\right)$ : a expectativa é de um efeito positivo sobre a quantidade exportada pelo país $i$, já que esta variável representa o tamanho econômico do país importador e, consequentemente, sua demanda por produtos e seu poder de compra.

Distância entre países $\left(D_{i j}\right)$ : Almeida e Silva (2006) afirmaram que a expectativa é de um coeficiente negativo para esta variável, já que a mesma representa uma resistência ao fluxo do comércio entre dois países.

PIB per capita do país importador $\left(m_{j}\right)$ : Maddison (1983) apresenta em seu trabalho dados históricos de PIB per capita de países desenvolvidos e de países em desenvolvimento (incluindo o Brasil). Vê-se que para o segundo grupo esses valores são sempre menores em relação aos do primeiro, portanto, esta variável foi utilizada como meio de representar o nível de industrialização do país importador. Espera-se que um país desenvolvido (maior $m_{j}$ ), produza papel a um custo mais baixo que aquele produzido no Brasil, restringindo a quantidade a ser importada. Portanto, para a exportação

Revista Árvore, Viçosa-MG, v.35, n.3, p.573-580, 2011 
de papel a expectativa é de um sinal negativo para esta variável. Em relação à celulose, espera-se sinal positivo já que quanto mais desenvolvida a nação, maior o consumo de papel e, consequentemente, maior necessidade de matéria prima para sua produção.

\subsection{Fonte de dados}

Foram empregados dados anuais, do período de 1997 a 2005, referentes ao produto interno bruto (PIB) e valores das exportações de papel e celulose do Brasil. Também foram obtidos dados sobre PIB e PIB per capita dos países importadores naquele período, bem como a distância geodésica desses países em relação ao Brasil. Ressalta-se que foram computados somente os dados de países que realizaram importações durante todos estes anos.

Assim, os países importadores considerados na análise do mercado de celulose foram: Argentina, Áustria, Canadá, China, Colômbia, França, Alemanha, Índia, Indonésia, Itália, Japão, Jordânia, Coréia do Sul, Espanha, Suíça, Tailândia, Turquia, Reino Unido, Estados Unidos, Uruguai, Venezuela e Vietnã. Na análise do mercado de papel, os países foram: Angola, Argentina, Austrália, Barein, Barbados, Bolívia, Camarões, Canadá, Chile, China, Colômbia, Costa Rica, Costa do Marfim, Cuba,

Chipre, República Dominicana, Equador, Egito, El Salvador, França, Alemanha, Guatemala, Guiné, Honduras, Israel, Itália, Jamaica, Japão, Coréia do Sul, Malásia, México, Holanda, Nova Zelândia, Omã, Paquistão, Panamá, Paraguai, Peru, Filipinas, Portugal, Senegal, Singapura, Espanha, Suriname, Suécia, Suíça, Trindade e Tobago, Tunísia, Reino Unido, Estados Unidos, Uruguai e Venezuela.

Os valores históricos de exportação de celulose e papel são da Food And Agriculture Organization (FAO, 2008). Os de PIB e PIB per capita, são do Serviço de
Investigação Econômica do United States Department of Agriculture (2008). As distâncias entre o Brasil e os demais países analisados foram obtidas no banco de dados do Centre D'etudes Prospectives et D'informations Internationales (CEPII, 2008).

\subsection{Testes estatísticos}

O grau de ajustamento das regressões foi avaliado por meio do coeficiente de determinação $\left(R^{2}\right)$. A significância das estimativas dos parâmetros foi verificada por meio do teste " $t$ ".

\section{RESULTADOS}

\subsection{Exportação de celulose}

Na Tabela 1 são apresentados os resultados obtidos com o ajuste do modelo de exportação de celulose pelo Brasil.

O coeficiente de determinação $\left(\mathrm{R}^{2}\right)$ indicou que $64,48 \%$ das variações ocorridas na exportação de celulose foram explicadas pelas variáveis presentes no modelo.

Para a variável PIB do Brasil $\left(M_{i}\right)$, o coeficiente foi significativo em nível de $6 \%$ de probabilidade enquanto os coeficientes das demais variáveis foram todos significativos em nível de $1 \%$ de probabilidade. Como as estimativas foram feitas a partir de um modelo logarítmico, os parâmetros podem ser interpretados como as estimativas das elasticidades.

\subsection{Exportação de papel}

O coeficiente de determinação $\left(\mathrm{R}^{2}\right)$ indicou que 61,26\% das variações ocorridas na exportação de celulose foram explicadas pelas variáveis presentes no modelo. Na Tabela 2 são apresentados os resultados obtidos com o ajuste da equação.

Tabela 1 - Estimativas do modelo de exportação de celulose pelo Brasil, 1997-2005.

Table 1 - Estimates of the Brazilian wood pulp export model, 1997-2005.

\begin{tabular}{|c|c|c|c|c|}
\hline Variáveis Explicativas & Descrição & Coeficiente Estimado & Erro-padrão & Teste " $t$ " \\
\hline & Constante & $-15,94130$ & & \\
\hline $\operatorname{Ln} M_{i}$ & PIB do Brasil & $4,4744^{*}$ & 0,9735 & 4,5961 \\
\hline $\operatorname{Ln} M_{j}$ & PIB do país importador & $0,2367 *$ & 0,0522 & 4,5312 \\
\hline $\operatorname{Ln} D_{i j}$ & Distância & $-0,8530 *$ & 0,1442 & $-5,9174$ \\
\hline Ln $m_{j}$ & PIB per capita do país importador & $-0,1077 * *$ & 0,0614 & $-1,7544$ \\
\hline
\end{tabular}

** significativo em nível de 8\%; * significativo em nível de $1 \%$.

Fonte: Resultados da pesquisa.

Revista Árvore, Viçosa-MG, v.35, n.3, p.573-580, 2011 
Tabela 2 - Estimativas do modelo de exportação de papel pelo Brasil, 1997-2005. Table 2 - Estimates of the Brazilian paper export model, 1997-2005.

\begin{tabular}{cccc}
\hline Variáveis Explicativas & Descrição & Coeficiente Estimado & Erro-padrão \\
\hline & Constante & $-15,94130$ & Teste “t” \\
Ln $M_{i}$ & PIB do Brasil & $4,4744^{*}$ & 0,9735 \\
Ln $M_{j}$ & PIB do país importador & $0,2367 *$ & 0,0522 \\
Ln $D_{i j}$ & Distância & $-0,8530^{*}$ & 0,1442 \\
Ln $m_{j}$ & PIB per capita do país importador & $-0,1077^{*}$ & 0,0614 \\
\hline $\mathrm{R}^{2}=0,6126$ & & & $-5,9174$ \\
\hline
\end{tabular}

** significativo em nível de 8\%; * significativo em nível de $1 \%$.

Fonte: Resultados da pesquisa.

O coeficiente para a variável PIB do per capita $\left(m_{j}\right)$, foi significativo em nível de $8 \%$ de probabilidade enquanto os coeficientes das demais variáveis foram todos significativos em nível de $1 \%$ de probabilidade.

\section{DISCUSSÃO}

Os coeficientes de determinação obtidos nos ajustes para expotação de celulose e de papel se apresentaram ligeiramente acima de $60 \%$, que é um desempenho aceitável em estudos com modelo gravitacional (CHENG; WALL, 1999; FEENSTRA et al., 2001; BAIER; BERGSTRAND, 2009).

No ajuste do modelo para exportação de celulose, o coeficiente da variável distância entre regiões apresentou sinal positivo igual a 0,4199, ao contrário do que se esperava. As demais variáveis presentes no modelo apresentaram-se com sinal positivo como esperado, indicando uma relação direta elas e a quantidade exportada pelo Brasil.

A elasticidade-PIB do Brasil foi 2,60, sugerindo que um aumento de $10 \%$ no PIB do país, ocasionaria um aumento de $26,0 \%$ na quantidade de celulose exportada, indicando que a exportação de celulose é elástica com relação ao PIB do Brasil.

Com relação ao PIB do país importador, pode-se dizer que um aumento de $10 \%$ no seu valor acarretaria um acréscimo de 5,01\% na quantidade de celulose exportada, enquanto para o PIB per capita esse acréscimo seria de $3,44 \%$.

Para a exportação de papel, os sinais de todos os coeficientes apresentaram-se como esperado.

A elasticidade-PIB do Brasil foi 4,47, sendo então que um aumento de $10 \%$ no PIB do país ocasionaria um aumento de $44,7 \%$ na quantidade de papel exportada, indicando que a exportação de papel é elástica com relação ao PIB do Brasil.
Para o PIB do país importador, vê-se que um aumento de $10 \%$ nessa variável acarretaria um acréscimo de 2,37\% na quantidade de papel exportada. Já para o PIB per capita, haveria um decréscimo de 1,08\% nas exportações caso este aumentasse $10 \%$.

Coerentemente, a variável Dij teve relação inversa com a quantidade exportada, indicando que uma diferença de 10\% na distância entre o Brasil e o país importador aumentaria $8,53 \%$ as exportações de papel.

Pensando no PIB per capita como indicador indireto de nível tecnológico de um país, vê-se que os mais industrializados importam menos papel do Brasil, enquanto os menos industrializados tendem a importar mais. O setor brasileiro de celulose e papel tem condições para continuar o crescimento acelerado e absorver cada vez mais, maior parcela de mercado por conta da alta produtividade de seus reflorestamentos (SOARES, 2007). Entretanto, apenas a vantagem absoluta obtida na matériaprima básica (cavaco de madeira) não consegue manter a competitividade internacional da indústria de papel, onde o padrão de concorrência é determinado pelas escalas de produção e o sucesso de um produtor de papel está estritamente relacionado à eficiência de sua tecnologia de produção. A pressão por baixos custos nesse ramo requer novos investimentos em tecnologia, normalmente associados ao processo e ao produto (FREUND, 1997; SILVA, 2003, 2004).

Apesar da distância não ter influenciado negativamente as exportações de celulose, para a exportação de papel ela foi fator de decréscimo do fluxo. O ocorrido indica alta competitividade da celulose brasileira no mercado externo. Barros e Goldenstein (1997) falam desta vantagem e dela ser fruto da alta produtividade das florestas (fonte de matéria prima) e do clima favorável. Aliados ao clima, os programas de melhoramento feitos ao longo das décadas permitiram ao Brasil ter nos dias de hoje clones de Eucalyptus 
de alta qualidade para produção de celulose kraft branqueada, alcançando os mais elevados níveis mundiais de incremento médio, em volume de madeira, por ano (GOMIDE et al., 2005).

O cenário encontrado no estudo pode ser observado nos gráficos apresentados na Figura 1. Mais da metade do papel exportado pelo Brasil vai para os países da América Latina, que são mais próximos e pouco desenvolvidos tecnologicamente. Já a celulose exportada vai em grande parte para países distantes (Ásia, Oceania e América do Norte). Valverde et al.(2006) cita que os maiores importadores de celulose do Brasil em 2003 foram EUA, Japão, Alemanha, China, Itália, França, Reino Unido e Bélgica.

O Brasil é o maior produtor de celulose de fibra curta a partir de eucalipto no mundo e está entre as maiores produtoras mundiais de papel (BRACELPA, 2009). O avanço e a manutenção dentro do setor irão depender do contínuo investimento estratégico demandado e, sobretudo, da eliminação de entraves econômicos envolvendo principalmente os custos e a estrutura do transporte no país, a burocracia operacional e a disponibilidade de energia, que acabam por frear a iniciativa empresarial no setor (RADICCHI, 2004; CNI, 2008).

\section{CONCLUSÕES}

O modelo apresentado tem êxito na predição das exportações de papel e celulose feitas pelo Brasil.

O PIB per capita dos países importadores age de maneira negativa sobre a quantidade de papel exportado e de maneira positiva sobre a quantidade de celulose exportada pelo Brasil.

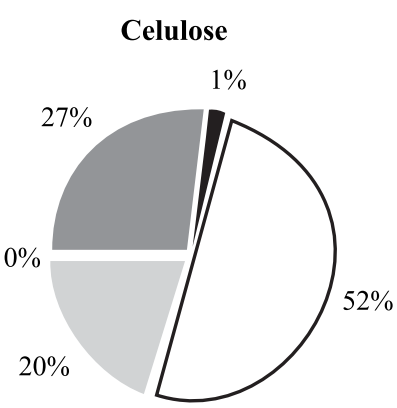

América do Norte África

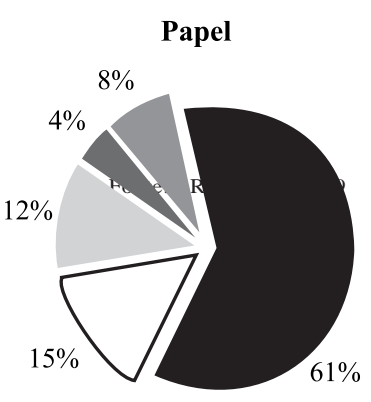

Ásia / Oceania
América Latina $\quad \square$ Europa

Figura 1 - Destino das exportações brasileiras em 2008. Figure 1 -Destination of Brazilian exports in 2008.
A exportação dos bens estudados varia diretamente com o PIB tanto do Brasil quanto dos países importadores.

As exportações, tanto de papel quanto de celulose, são mais sensíveis a variações no PIB do Brasil do que a variações no PIB, PIB per capita e na distância dos países importadores.

A distância não influencia negativamente as exportações da celulose brasileira, mas para a exportação de papel ela é fator de decréscimo.

\section{REFERÊNCIAS}

ALMEIDA, F. M.; SILVA, O. M. A guerra fiscal e o comércio interestadual brasileiro em uma análise setorial. Revista Economia e

Desenvolvimento, n.18, p.2-15, 2006.

ANDERSON, J. E. A theoretical foundation for the gravity equation. American Economic Review, v.69, n.1, p.106-116, 1979.

ASSOCIAÇÃO BRASILEIRA DE CELULOSE E PAPEL - BRACELPA. Desempenho do setor e projeções. 2009.

Disponível em: <http:// www.bracelpa.org.br $>$. Acesso em: 20 maio 2009.

BAIER, S. L.; BERGSTRAND, J. H. Bonus vetus OLS: A simple method for approximating international trade-cost effects using the gravity equation. Journal of International Economics, v.77, n.1, p.77-85, 2009.

BARROS, J. R. M.; GOLDENSTEIN, L. Avaliação do processo de reestruturação industrial brasileiro. Revista de Economia Política, v.17, n.2, p.10-31, 1997.

BERGSTRAND, J. H. The gravity equation in international trade: some microeconomic foundations and empirical evidence. The Review of Economics and Statistics, v.67, n.3, p.474-481, 1985.

BERGSTRAND, J. H. The generalized gravity equation, monopolistic competition, and the factor-proportions theory in international trade. The Review of Economics and Statistics, v.71, n.1, p.143-153, 1989. 
CENTRE D’ETUDES PROSPECTIVES ET D'INFORMATIONS INTERNATIONALES - CEPII. Databases - Distance. Disponível em:<http:/ /www.cepii.fr/anglaisgraph/bdd/distances.htm>. Acesso em: 01 julho 2008.

CHENG, I.; WALL, H. Controlling for heterogeneity in gravity models of trade. The Federal Reserve Bank of Saint Louis Working Paper, n.99-010A, 1999.

CONFEDERAÇÃO NACIONAL DAS INDÚSTRIAS - CNI. Pesquisa: Os problemas da empresa exportadora brasileira: 2008 / CNI. Brasília: 2008. 130p.

DEARDORFF, A. Determinants of bilateral trade: does gravity work in a neo-classical world? In: FRANKEL, J. (Ed). The Regionalization of the World Economy. Chicago: University of Chicago Press, 1998. p.7-31.

EVENETT, S. J.; KELLER, W. On theories explaining the success of the gravity equation. Journal of Political Economy, v.110, n.2, p.281-316, 2002.

FEENSTRA, R. C.; MARKUSEN, J. A.; ROSE, A. K. Using the gravity equation to differentiate among alternative theories of trade. Canadian Journal of Economics, n.34, v.2, p.430-447, 2001.

FOOD AND AGRICULTURE ORGANIZATION OF THE UNITED NATIONS - FAO. Forestry trade flow (Indicative Raw Data). Disponível em:<http://faostat.fao.org/site/382/default.aspx>. Acesso em: 23 jun. 2008.

FRANKEL, J. Regional trading blocs in the world economic system.

Washington, D.C.: Institute for International Economics, 1997. 388p.

FREUND, O. Mercados papeleiros de lento crescimento representam 80\% do consumo mundial. Entrevistadora: Patrícia Capo. O Papel, v.58, n.8, p.45-53, 1997.

GOMIDE, J. L. et al Caracterização tecnológica para produção de celulose, da nova geração de clones de Eucalyptus do Brasil. Revista Árvore, v.299, n.1, p.129-137, 2005.

MADDISON, A. A comparison of levels of GDP per capita in developed and developing countries, 1700-1980. The Journal of Economic History, v.43, n.1, p.27-41, 1983.
BRASIL. Ministério do Desenvolvimento, Indústria e Comércio Exterior. Balança Comercial dados consolidados de janeiro a dezembro de 2008. Disponível em: <http:// www.desenvolvimento.gov.br/arquivos/ dwnl_1235161747.pdf>. Acesso em: 27 maio 2009.

MORAES, R. Papel e celulose: Partida de novas máquinas garante crescimento maior da produção nacional em 2008, adotando as melhores tecnologias disponíveis. Revista Química e Derivados, n.469, 2008. Disponível em:<http:// www.quimicaederivados.com.br/revista/qd469/ papel-celulose.html>. Acesso em: 30 jun. 2008.

RADICCHI, C. C. Competitividade das exportações brasileiras de celulose: uma análise do custo Brasil. 2004. 83f. Dissertação (Mestrado em Economia Aplicada) - Universidade Federal de Viçosa, Viçosa, MG, 2004.

SILVA, C. L. Gestão da exportação da indústria brasileira de papel de imprimir e escrever: uma análise sob a ótica da cadeia de valor. In: ENANPAD ENCONTRO DO ANPAD, 28., 2003, Atibaia. Anais ... Atibaia: ANPAD, 2003. v.1. p.210-226.

SILVA, C. L. Fatores determinantes da competitividade internacional da indústria de papel de imprimir e escrever sob a ótica da cadeia de valor. Revista Brasileira de Gestão de Negócios, v.6, n.14, p.42-59, 2004.

SILVA, O. M.; ALMEIDA, F. M.; OLIVEIRA, B. M. Comércio internacional “ $\mathrm{x}$ ” intranacional no Brasil: medindo o efeito-fronteira. Nova

Economia, v.17, n.3, 2007.

SOARES, N. S.; SILVA, M. L.; LIMA, J. E. A função de produção da indústria brasileira de celulose, em 2004. Revista Árvore, v.31, n.3, p.495-502, 2007.

SOUZA, R. L.; OLIVEIRA, M. J. L. Desempenho da Indústria de Papel e Celulose de Mercado: Brasil e Bahia - 1999 / 2001. Conjuntura \& Planejamento, n.92, p.19-29, 2002.

UNITED STATES DEPARTMENT OF AGRICULTURE - USDA. International Macroeconomic Data Set. Disponível em:<http://www.ers.usda.gov/Data/ Macroeconomics/>. Acesso em: 23 jun. 2008.

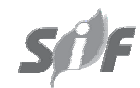

Revista Árvore, Viçosa-MG, v.35, n.3, p.573-580, 2011 
VALVERDE, S. R.; SOARES, N. S.; SILVA, M. L.

Desempenho das exportações brasileiras de celulose. Revista Árvore, v.30, n.6, p.1017-1023, 2006.
VIEIRA, L. A. N. et al. Dimensionamento do setor florestal em Minas Gerais. Cerne, v.12, n.4, p.389-398, 2006. 